Original Article

\title{
Retinopathy of prematurity: Prevalence and risk factors
}

\author{
Rinawati Rohsiswatmo
}

\begin{abstract}
Background Retinopathy of prematurity (ROP) is one of the major causes of infant blindness. There are several factors known as risk factors for ROP. Recent studies show ROP as a disease of multifactorial origin.

Objective To report the prevalence of ROP in Cipto Mangunkusumo Hospital, Jakarta and its relation to several risk factors.

Methods A cross-sectional descriptive study was conducted from December 2003-May 2005. All infants with birth weight 2500 grams or less, or gestational age 37 weeks or less, were enrolled consecutively and underwent the screening of ROP at 4 to 6 weeks of chronological age or 31 to 33 weeks of postconceptional age.

Result Of 73 infant who met the inclusion criteria, 26\% (19 out of 73 infant) had ROP in various degrees. About $36.8 \%$ (7 out of 19 infants) were in stage III or more/threshold ROP. No ROP was noted in infants born $>35$ weeks of gestational age, and birth weight $>2100$ grams. No severe ROP was found in gestational age $>34$ weeks and birth weight $>1600$ grams. None of full-term, small for gestational age infants experienced ROP. Birth weight, sepsis, apneu, asphyxia, multiple blood transfusions, and oxygen therapy for more than 7 days were statistically significant with the development of ROP. However, using multivariate analysis, only asphyxia, multiple blood transfusions, and oxygen therapy for more than 7 days were statistically significant with the development of ROP.

Conclusion Screening of ROP should be performed in infants born 34 weeks of gestational age and/or birth weight $<1600$ grams. Infants with birth weight from 1600- $\leq 2100$ grams need to be screened only if supplemental oxygen is necessary or with clinically severe illness [Pediatr Indones 2005;45:270-274].
\end{abstract}

Keywords: retinopathy of prematurity, screening, risk factors
A

$s$ advances in neonatology develop, the survival of premature and lower birth weight babies increases, consequently the frequency of retinopathy of prematurity (ROP) will rise. ${ }^{1,2}$ Retinopathy of prematurity is a vasoproliferative disorder of the eye affecting premature neonates. ${ }^{3}$ Most cases resolve spontaneously, but in its more severe forms, it may result in severe visual impairment or blindness. ${ }^{3-5}$

Literature identifiies numerous potential risk factors for ROP, revealing that it is a multifactorial disease. ${ }^{1}$ These factors include birth weight, gestational age, sepsis, respiratory distress, apneu, asphyxia, small for gestational age (SGA), multiple blood transfusions, prolonged supplemental oxygen, and unstable oxygen saturation levels. ${ }^{1-3,6,7}$ Although many causative factors have been proposed for ROP, only birth weight, gestational age, and supplemental oxygen therapy is consistently associated with the disease. ${ }^{3}$

The lower an infant's birth weight and gestational age at birth, the more likely they are to develop ROP. The cryotherapy for retinopathy of prematurity (CRYO-ROP) group found that $47 \%$ of infants with birth weight from 1000-1250 grams have some de-

From the Department of Child Health, Medical School, University of Indonesia, Jakarta, Indonesia.

Reprint requests to: Rinawati Rohsiswatmo, MD, Department of Child Health, Medical School, University of Indonesia, Cipto Mangunkusumo Hospital, Jl. Salemba Raya 6, Jakarta 10430. Tel. 62-21-3146811, Fax: 62-21-3907743. 
gree of ROP, as compared with $90 \%$ of infants with birth weight less than 750 grams. A similar pattern is seen for gestational age, ROP occurring in 30\% of infants born at more than 31 weeks gestation and in $83 \%$ of infants born at less than 28 weeks gestation. ${ }^{8}$

The awareness for screening and treatment for ROP has increased among both pediatricians and ophtalmologists since the last five years. However, in Indonesia there are no published reports of ROP and a screening guideline has not been established yet. Actually local criteria for each regional population and neonatal unit are required to determine the screening guideline. ${ }^{4}$ Some less developed countries found that bigger, more mature infants develop threshold/ severe ROP. ${ }^{9}$ In the Neonatology Division of Cipto Mangunkusumo Hospital, the screening of ROP has been performed routinely since December 2003 and we have found a case of ROP in an infant with birth weight 2500 grams and gestational age of 37 weeks who experienced severe sepsis. Consequently, the screening guideline in our unit is infants with birth weight 2500 grams or less and/or gestational age 37 weeks or less.

The aim of this study is to recognize the prevalence of ROP and its relation to several risk factors.

\section{Methods}

A cross-sectional descriptive study was conducted in the Neonatal Ward at Cipto Mangunkusumo Hospital from December 2003-May 2005. Inclusion criteria were infants with birth weight 2500 grams or less and/ or gestational age of 37 weeks or less who underwent ROP screening. Gestational age was assessed as completed weeks using obstetric estimates based on the date of the last menstrual period of the mother and confirmed by Ballard Method. The subjects were recruited consecutively.

The ophthalmic examination was initially performed by vitreoretina ophtalmologists at 4 to 6 weeks of chronological age or 31 to 33 weeks of postconceptional age. If no ROP was found, eye examinations were continued every one or two weeks until complete vascularization of retina, death, or discharge. Diagnosis and stage of ROP was classified by vitreoretina ophtalmologists according to International Classification of Retinopathy of Prematurity.
Risk factors related with ROP were recorded, such as birth weight, gestational age, sepsis, respiratory distress (not caused by sepsis), small for gestational age, asphyxia, apnea, multiple blood transfusions, and duration of oxygen therapy. The correlation between the stages of ROP with birth weight and gestational age was processed by Epi-Info.

\section{Results}

During the study period, 73 infants were enrolled and underwent ROP screening examination. The gestational age ranged from 27 to 40 weeks (median 33 weeks), birth weight ranged from 950 to 2500 grams (median 1600 grams). Three infants were full-term SGA infants, and 12 preterm SGA infants. There were 28 male infants and 45 female infants. Forty eight infants received oxygen therapy for more than 7 days, 20 infants less than 7 days, and 5 infants did not receive oxygen. Subject characteristics are shown in Table 1. No ROP was noted in five infants who did not receive oxygen. They were preterm infants, with gestational age (GA) of 33-36 weeks, birth weight (BW) from 1440-2120 grams, and only one infant (GA 36 weeks, BW 2120 grams) experienced sepsis.

The prevalence of ROP was 26\% (19 out of 73 patients). The prevalence of ROP infants with birth weight 1500 grams or less was $19.2 \%$ (14 out of 73 patients), gestational age 32 weeks or less was $16.4 \%$ (12 out of 73 patients). Threshold ROP (severe ROP/ stage III or more) was found in 7 infants (9.6\%). Five infants with birth weight less than 1500 grams and

TABle 1. Subject Characteristics

\begin{tabular}{lll}
\hline Subject characteristics & median \pm SD & $\mathbf{n}$ \\
\hline Gestational age (weeks) & $33 \pm 2.7$ & \\
Birth weight (grams) & $1600 \pm 374$ & \\
Sex & & 28 \\
$\quad$ Male & 45 \\
$\quad$ Female & \\
Small for gestational age & 3 \\
$\quad$ Full-term baby & 12 \\
$\quad$ Preterm baby & \\
Duration of oxygen therapy & 5 \\
$\quad$ None & 20 \\
$\quad<7$ days & 48 \\
$\quad \geq 7$ days & \\
\hline
\end{tabular}


gestational age 32 weeks or less experienced threshold ROP. None of full-term SGA infants experienced ROP, compared with three of preterm SGA infants experienced ROP.

The birth weight groups ranged from 950 to 2100 grams (median: $1300 \pm 271.7$ grams) and the gestational age ranged from 27 to 35 weeks (median: $32 \pm$ 2.4 weeks). The non-ROP group had birth weight from 1000 to 2500 grams (median: $1700 \pm 354.5$ grams), the gestational age ranged from 28 to 40 weeks (median: $34 \pm 2.5$ weeks). Distribution of ROP and non$\mathrm{ROP}$ group in relation with birth weight and gestational age is shown in Table 2. Lower birth weight and younger gestational age were statistically significant in the development of ROP.

Severe ROP infants have birth weight from 1100 to 1600 grams (median: $1240 \pm 210.4$ grams) and gestational age from 27 to 34 weeks (median: $30 \pm 2.6$ weeks).

Stages of ROP was not correlated with lower birth weight PR 2.86 (CI 95\% 0.96;8.47) and younger gestational age PR 1.71 (CI 95\% 0.61;4.78) (Table 3).

Birth weight PR 6.650 (CI 95\% 2.051; 21.563), sepsis PR 1.102 (CI 95\% 1.012; 1.200), apneu PR 6.375 (CI 95\% 1.857; 21.887), asphyxia PR 3.917 (CI 95\% 1.1151; 13.326), multiple blood transfusions PR 4.350 (CI 95\% 1.277; 14.820), and oxygen therapy for more than 7 days PR 0.159 (CI 95\% 0.033; 0.756) were statistically significant with the development of ROP. However, from multivariate analysis alone, asphyxia, multiple blood transfusions, and oxygen therapy for more than 7 days were statistically significant with the development of ROP.

TABle 2. Distribution of ROP AND NON-ROP GROUP RELATED WITH BIRTH WEIGHT AND GESTATIONAL AGE

\begin{tabular}{lcc}
\hline & \multicolumn{2}{c}{ ROP (n) } \\
\cline { 2 - 3 } & $\mathbf{( + )}$ & $\mathbf{( - )}$ \\
\hline Birth weight (grams) & 1 & 1 \\
$\quad<1000$ & 13 & 15 \\
$1000-1500$ & 4 & 28 \\
$1501-2000$ & 1 & 10 \\
$2001-2500$ & & \\
Gestational age (weeks) & 2 & 0 \\
$\leq 28$ & 10 & 22 \\
$29-32$ & 1 & 13 \\
$33-34$ & 0 & 3 \\
$35 \leq \mathrm{GA} \leq 40$ & &
\end{tabular}

\section{Discussion}

The World Health Organization's Vision 2020 program targets $\mathrm{ROP}$ as an avoidable disease requiring early detection and treatment to prevent blindness. These strategies have successfully reduced the incidence of ROP, such as, routine fundus examination of premature neonates at less than 32 weeks gestation or under 1250 grams birth weight, provision of carefully monitored levels of supplemental oxygen, where necessary, and treatment by well trained and well equipped ophtalmologists. ${ }^{3}$

Cryotherapy for retinopathy of prematurity multicenter study (1989-1997) reported that the incidence of ROP was 21.3\% (202 out of 950 infants with gestational age less than 37 weeks). ${ }^{10}$ Our study revealed that the prevalence of ROP was 26\% (19 out of 73 patients) and the threshold RPP was $9.6 \%$ (7 out of 73 infants). The result was almost the same with Yang et $a l^{2}$ in Taiwan 25\% (27 out of 108 patients with birth weight less than 2000 grams or gestational age less than 36 weeks) and the threshold $\mathrm{ROP}$ was $7 \%$.

In 2001, the American Academy of Pediatrics and the American Academy of Ophthamology have recommended screening in infants with birth weight less than 1500 grams and gestational age less than 28 weeks, with or without supplemental oxygen; or in clinically severe illness infants with birth weight 1500 to 2000 grams. ${ }^{11,12}$ Gilbert et $a l^{9}$ showed that infants who develop severe ROP in highly developed countries differ from those who are affected in less well developed countries. Consequently, the screening guidelines are different in each country. In highly developed countries such as United States and United Kingdom, examinations are done in infants with birth weight less than 1500 grams or gestational age less

Table 3. The correlation between the stages of ROP WITH THE BIRTH WEIGHT AND GESTATIONAL AGE

\begin{tabular}{ccc}
\hline Stages of ROP & $\begin{array}{c}\text { Birth weight } \\
\text { (median } \pm \text { SD) } \\
\text { grams }\end{array}$ & $\begin{array}{c}\text { Gestational age } \\
\text { (median } \pm \text { SD) } \\
\text { weeks }\end{array}$ \\
\hline $0(n=1)$ & 1200 & 33 \\
$1(n=8)$ & $1400 \pm 334.6$ & $32.5 \pm 2.2$ \\
$2(n=3)$ & $1320 \pm 280.4$ & $32 \pm 2.3$ \\
$\leq 3(n=7)$ & $1240 \pm 210.4$ & $30 \pm 2.6$ \\
\hline
\end{tabular}


than 32 weeks. However, some less developed countries widen their screening criteria, because bigger, more mature infants develop threshold ROP, for instance, India examines infants with birth weight less than 2000 grams or gestational age less than 35 weeks. ${ }^{9}$ Our inclusion criteria for screening were different from other centers because yet there are no baseline data in Indonesia.

The CRYO-ROP multicenter study reported no ROP was noted in infants born at more than 32 weeks, and no severe ROP was noted in infants at more than 28 weeks of gestational age. ${ }^{10}$ In our study, no ROP was noted in infants born at more than 35 weeks of gestational age and birth weight more than 2100 grams. No severe ROP was found in gestational age more than 34 weeks and birth weight more than 1600 grams. The discrepancy might be due to the differences in race and in systems applied by the neonatal unit of population studied. Our study, showed regional criteria for $\mathrm{ROP}$ screening is required. In the following year, our screening guideline may be altered to screen infants born at $\leq 34$ weeks of gestational age and/or birth weight $\leq 1600$ grams. Infants born with birth weight from $1600-\leq 2100$ grams need to be screened only if supplemental oxygen is necessary; or in clinically severe illness with birth weight less than 2200 grams and/or gestational age less than 36 weeks. The guideline should be evaluated and modified periodically based on the evolving knowledge and advancements in neonatal care unit. ${ }^{4}$

The pathogenesis of the development of ROP is still uncertain. Recent observation has found ROP to be a disease of multifactorial origin., 1,6 The altered regulation of vascular endothelial growth factor (VEGF) has been suggested as one of the factors in the pathogenesis of ROP. In premature infants, the retina is incompletely vascularized. After premature birth, relative hyperoxia may downregulate VEGF production. Administration of supplemental oxygen may lead to sustained hyperoxia, setting the stage for vaso-obliteration of existing vessels and arrest of the vascularization. Over time, as the metabolic demands of the developing eye increases, the immature nonperfused area of the retina becomes hypoxic and may overproduce VEGF pathologically. High levels of VEGF stimulate neovascularization of the retina, which in severe cases may result in retinal fibrosis dan retinal detachment. . $^{2,13}$
Sepsis in the low birth weight premature infants is frequently accompanied with hypotension which will impair tissue perfusion with local hypoxia to render retina under the risk of ROP development. Respiratory distress syndrome was known to be associated with systemic hypoxia, which will lead to retina ischemia. $^{2}$

Chronic hypoxia in utero and intrauterine growth retardation are two prenatal conditions that seem to be related to the development of ROP.,8 In the current, study full-term SGA infants who received supplemental oxygen did not develop ROP, compared with 3 in 12 preterm SGA infants who did. Our study encouraged that in the low birth weight full-term infants it is unnecessary to undergo ROP screening.

It is hypothesized that the damaging effects of blood transfusions on the retina are mediated by an increase in free iron. Plasma non-transferrin boundfree iron was significantly increased in preterm infants after blood transfusion, but was not observed in fullterm infants after blood transfusion. ${ }^{6,14}$

High concentrations of oxygen therapy was previously thought as the major factor for the development of ROP. ${ }^{2}$ However, some reports have found ROP may develop in premature infants who have received little or no supplemental oxygen. Even after oxygen therapy, not all premature infants develop ROP. These evidences suggested that risk factors other than oxygen might play an important role in the development of $\mathrm{ROP}^{3}$

As mentioned above, the repeated episodes of hyperoxia and hypoxia is important factor in the development of ROP. An oxygen management policy has to be implemented in the neonatal unit, such as a strict guideline in the practices of increasing and weaning fraction of inspired oxygen $\left(\mathrm{FiO}_{2}\right)$ and the monitoring of oxygen saturation $\left(\mathrm{SpO}_{2}\right)^{2} \cdot 1,3$ In our study, we only recorded the duration of oxygen therapy because monitoring of $\mathrm{SpO}_{2}$ just could have been implemented in our unit since 2004. Our findings urged us to make strict guidelines in the practice of increasing or weaning of oxygen therapy and the monitoring of oxygen saturation. The guideline includes curtail unnecessary $\mathrm{O}_{2}$ administration, prevent abrupt changes in $\mathrm{O}_{2}$ saturation, maintain certain $\mathrm{O}_{2}$ saturation according to gestational age. ${ }^{5,14}$

Birth weight, gestational age, duration of ventilation therapy, blood transfusion, respiratory distress 
syndrome, and sepsis were reported previously to be associated with increased risk of ROP (as citated from Ikeda). ${ }^{6}$ Yang et al ${ }^{2}$ reported low birth weight and young gestational age are the most important risk factors in the development of ROP. Our study showed that birth weight, sepsis, apneu, asphyxia, multiple blood transfusions, and oxygen therapy of more than 7 days were statistically significant with the development of ROP. However, using multivariate analysis, only asphyxia, multiple blood transfusions and oxygen therapy for more than 7 days were statistically significant with the development of ROP.

Yang et $\mathrm{al}^{2}$ showed that there was a correlation between severity of ROP with lower birth weight and younger gestational age. It was different from our study, may be because survival of extremely preterm infants and extremely very low birth weight in our unit was low.

We concluded that asphyxia, multiple blood transfusions, and oxygen therapy for more than 7 days are the most important risk factors in the development of ROP. The screening guideline should be evaluated and modified periodically based on the evolving knowledge and the advancements in neonatal care unit. The low birth weight full-term infants should not necessarily undergo ROP screening.

\section{References}

1. Schaffer DB, Palmer EA, Plotsky DF, Metz HS, Flynn JT, Tung B, et al. Prognostic factors in the natural course of retinopathy of prematurity. Ophtalmol 1993;100: 230-7.

2. Yang C-S, Chen S-J, Lee F-L, Hsu W-M, Liu J-H. Retinopathy of prematurity, screening, incidence, and risk factors analysis. Chin Med J 2001;64:706-12.

3. Wheatley CM, Dickinson JL, Mackey DA, Craig JE, Sale MM. Retinopathy of prematurity, recent advances in our understanding. Br J Ophtalmol 2002;86:696701.
4. Trinavarat A, Atchaneeyasakul L, Udompunturak S. Applicability of American and British criteria for screening of the retinopathy of prematurity in Thailand. Jpn J Ophtalmol 2004:48:50-3.

5. McNamara JA, Moreno R, Tasman WS. Retinopathy of prematurity. In: Tasman WS, Jaeger EA, editors. Duane's clinical ophtalmology. Vol 3. Philadelphia: Lippincott-Raven; 1997. p. 1-18.

6. Ikeda H, Kuriyama S. Risk factors for retinopathy of prematurity requiring photocoagulation. Jpn J Ophtalmol 2004;48:68-71.

7. Allegaert K, Verdonck N, Vanhole C, deHalleux V, Nauhalers G, Cossey V, et al. Incidence, perinatal risk factors, visual outcome and management of threshold retinopathy. Bull Soc belge Ophtalmol 2003;287:37-42.

8. Retinopathy of prematurity. Available from: URL: http://www. mrcoptham

9. Gilbert C, Fielder A, Gordillo L, Quinn G, Semiglia R, Visintin $\mathrm{P}$, et al. Characteristics of infants with severe retinopathy in countries with low, moderate, and high levels of development, implications for screening programs. Pediatrics 2005;115:e518-25.

10. Hussain N, Cliva J, Bhandari V. Current incidence of retinopathy of prematurity, 1989-1997. Available from: URL: http//:www.pediatrics.org/cgi/content/ full/104/3/e26.

11. Andruscavage L, Weissgold DJ. Screening for retinopathy of prematurity. Br J Ophtalmol 2002;86:1127-30.

12. American Academy of Pediatrics, the American Association of Pediatric Ophtalmology and Strabismus, the American Academy of Ophtalmology. Screening examination of premature infants for retinopathy of prematurity. Pediatrics 2001;108:809-11.

13. Chow LC, Wright KW, Sola A. Can changes in clinical practice decrease the incidence of severe retinopathy of prematurity in very low birth weight infants. Pediatrics 2003;111:339-45.

14. Tin W, Milligan DWA, Pennefather P, Hey E. Pulse oxymetry, severe retinopathy, and outcome at one year in babies of less than 28 weeks gestation. Arch Dis Child Fetal Neonatal 2001;84:F106-10. 